\title{
Estudio técnico y económico del tomate tipo cereza élite (Solanum lycopersicum L. var. cerasiforme) bajo condiciones semicontroladas
}

\section{Economic study of the elite cherry tomato type (Solanum lycopersicum L. var. cerasiforme) under semicontrolled conditions}

HERMAN DE JESÚS HERRERA'

ALEJANDRO HURTADO-SALAZAR ${ }^{2}$

NELSON CEBALLOS-AGUIRRE ${ }^{3,4}$

Planta de tomate tipo cereza con frutos maduros de la introducción élite IAC1624.

Foto: N. Ceballos-Aguirre

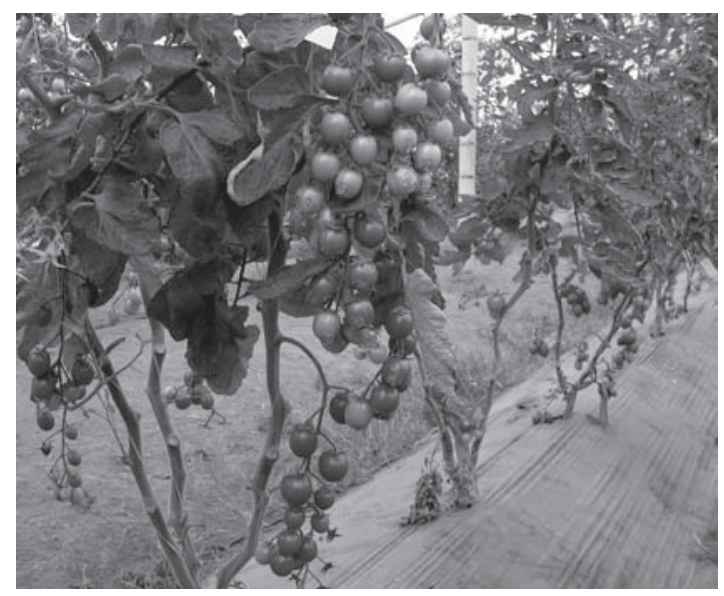

\section{RESUMEN}

El tomate tipo cereza por ser un cultivo de rápido retorno económico viene despertando el interés de los productores, pero aún hay poca información sobre su estructura de costos de producción y rentabilidad en las condiciones del país, impidiendo la expansión del cultivo. El objetivo del presente estudio fue evaluar técnica y económicamente diez introducciones élites de tomate tipo cereza, bajo condiciones semicontroladas en la región andina colombiana. El ensayo se realizó en la granja Tesorito, Manizales, Colombia. Se estableció un diseño experimental en bloques completos al azar con 10 tratamientos (introducciones élites) y un testigo comercial (Sweet million), con cuatro repeticiones. Las variables fisiológicas evaluadas fueron: rendimiento (g/planta), peso de frutos perdidos, rendimiento neto, rendimiento en $\mathrm{kg} \mathrm{ha}^{-1}$, número de frutos por racimo, número de racimos por planta, peso promedio de fruto, y como variables económicas se estimaron los costos de producción, el margen unitario de producción, la relación beneficio/costo, la tasa de retorno y se realizó un análisis de sensibilidad. Los resultados muestran que las introducciones IAC391, IAC1621 e IAC1688 para las condiciones del estudio superan las perspectivas financieras, convirtiendo estos materiales en opciones para los productores.

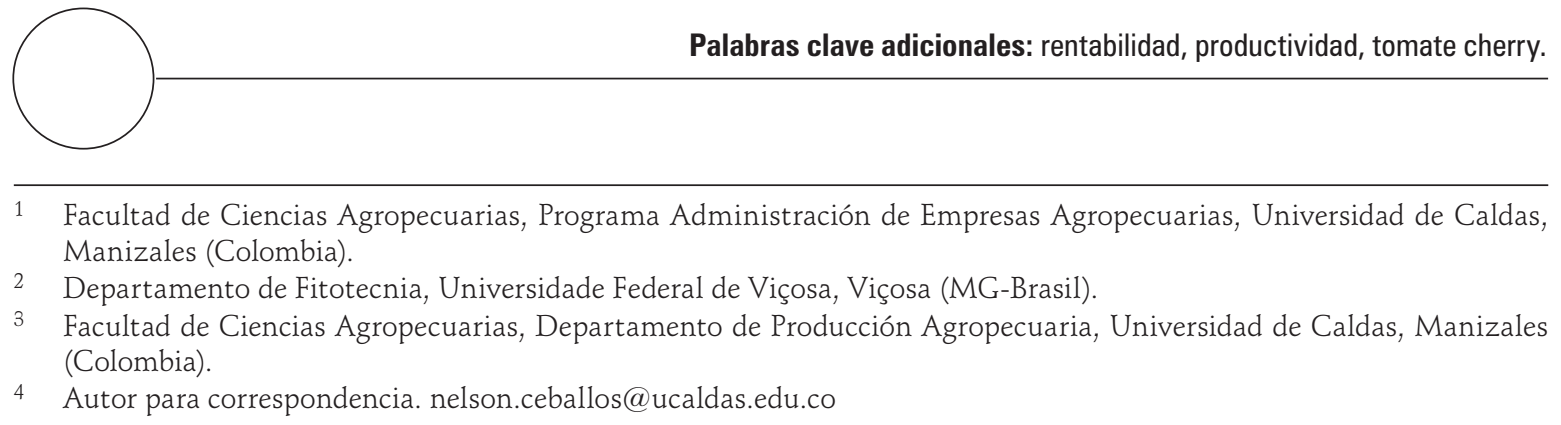




\section{ABSTRACT}

The cherry tomato, being a crop of fast economic return, has aroused the interest of producers, but there is little information on the costs of production and profitability in the domestic market, which prevents crop expansion. Thus, the objective of this study was to evaluate 10 economically elite introductions of cherry tomato under controlled conditions in the Colombian Andes. The study was conducted on the Tesorito Farm owned by Caldas University located in the city of Manizales (Colombia). The experimental design used completely randomized blocks with 10 treatments (elite introductions) and a commercial control (Sweet million), with four repetitions. The physiological variables were: yield (g/plant), weight of lost fruits, net yield, yield in $\mathrm{kg} \mathrm{ha}^{-1}$, number of fruits per cluster, number of bunches per plant, and average fruit weight. The following economic variables were estimated: production costs, production unit margin, cost benefit ratio, and rate of return; in addition, a sensitivity analysis was performed. The results showed that the IAC 391, IAC 1621 and IAC 1688 introductions exceeded the financial perspective under the studied conditions, making these materials options for producers.

Additional key words: profitability, productivity, cherry tomato.

Fecha de recepción: 16-07-2015

Aprobado para publicación: 20-11-2015

INTRODUCCIÓN

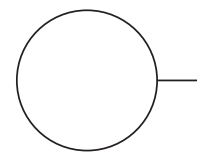

En la actualidad el cultivo de tomate (Solanum lycopersicum L.) es una de las hortalizas más plantadas en el mundo (Bai y Lindhout, 2007). La demanda de este producto aumenta continuamente e incentiva a los diferentes eslabones de la cadena productiva a su adopción, por lo que representa uno de los cultivos hortícolas de mayor repercusión socioeconómica a nivel mundial (Testa et al., 2014). En 2012 el área de tomate en el mundo alcanzó las 4,8 millones de hectáreas, y entre los años 2003 y 2012 presentó un aumento del 17,3\%, alcanzando rendimientos promedios de $24.800 \mathrm{~kg} \mathrm{ha}^{-1}$ (FAOSTAT, 2013). De acuerdo con Testa et al. (2014), ese incremento se debe al incremento del área sembrada en África $(+34,9 \%)$ y Asia (+30,8\%), mismos que para 2012 representaron el $21 \%$ y el $58 \%$ de las áreas de tomate en el mundo; mientras Europa $(-23,8 \%)$ y América (-11,6\%) presentaron disminuciones significativas con respecto al área dedicada al cultivo en 2003. Se destaca que las nuevas áreas de tomate corresponden a países y áreas productivas nuevas, en detrimento de los países y áreas tradicionales del cultivo. Esta tendencia puede deberse no solo a los bajos costos de la mano de obra y por las continuas inversiones que, en los últimos años, han afectado las técnicas de cultivo, sino también a las estrategias comerciales y de mercadeo. Por su parte, para Colombia se reportaron en 20134.160 ha que aportaron 175.076 t cosechadas (Encuesta Nacional Agropecuaria, 2014), lo que representa una participación baja a nivel mundial.

De acuerdo con Perilla et al. (2011), en los últimos años Colombia se ha caracterizado por un buen ritmo de crecimiento como resultado del mejoramiento de los circuitos comerciales y la tecnificación de los cultivos, se ve representada en la producción bajo invernadero, mecanismo de manejo integrado de plagas, implementación de buenas prácticas agrícolas, manejo de los estándares fitosanitarios para la exportación. El cultivo del tomate se está desarrollando, cada día más, bajo sistemas controlados y semicontrolados con el fin de evadir el riesgo de factores climáticos adversos 
(Gómez-Duque, 2010), estos factores externos pueden afectar positiva o negativamente el rendimiento, la calidad del fruto, la susceptibilidad a insectos y enfermedades. Uno de los problemas actuales en Colombia es la carencia de: cultivares nacionales, tecnologías adecuadas para la producción y el manejo poscosecha, y altos costos de producción que hacen del cultivo del tomate sea una actividad poco competitiva y sostenible.

Perilla et al. (2011) detectaron una importante demanda del tomate producido bajo cubierta tanto en el mercado nacional como internacional. De este modo surge como alternativa el desarrollo de nuevos cultivares nacionales de tomate tipo cereza, aprovechando el potencial genético por ser parte del centro de origen del mismo (región andina de Chile, Bolivia, Peru, Ecuador y Colombia), presentando unas formas silvestres promisorias como L. esculentum var. Cerasiforme y $L$. pimpinellifolium, estos materiales tienen un alto potencial por su alta variabilidad genética para programas de mejoramiento y como alternativa productiva para la región.

El objetivo del presente estudio fue evaluar económicamente 10 materiales élites promisorios de tomate tipo cereza (Solanum lycopersicum L. var. Cerasiforme) seleccionados previamente por caracteres de rendimiento y calidad del fruto, bajo condiciones semicontroladas en la región andina colombiana.

\section{MATERIALES Y MÉTODOS}

El estudio se realizó en la Granja Tesorito de la Universidad de Caldas, localizada en el municipio de Manizales, departamento de Caldas (Colombia); con altitud de $2.340 \mathrm{msnm}$, temperatura media anual de $17,5^{\circ} \mathrm{C}$, humedad relativa del $78 \%$, precipitación anual de $2.000 \mathrm{~mm}$, brillo

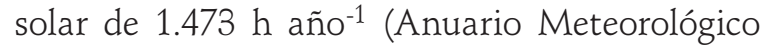
Cafetero 2012 y 2013) y andisoles derivados de cenizas volcánicas con textura franco arenosa ricos en materia orgánica.
Los materiales evaluados fueron 10 introducciones de tomate tipo cereza élite: IAC391, IAC1624, IAC426, IAC424, IAC1688, IAC 1621, LA 2076, LA 2692, LA445 y LA412, previamente seleccionados por su rendimiento y calidad del fruto. Como testigo se utilizó el hibrido Sweet million.

Los ensayos se establecieron bajo condiciones semicontroladas con una cubierta plástica tipo Agroclear ${ }^{\circledR}$ y en el suelo con cobertura plástica tipo Agromoulch $\mathrm{X}^{\circledR}$ (negro/negro) en dos módulos de invernaderos de $12 \times 40 \mathrm{~m}\left(480 \mathrm{~m}^{2}\right)$ en "capilla a dos aguas" con una estructura hecha en guadua, en un área total de $960 \mathrm{~m}^{2}$. Las distancias de siembra utilizadas fueron $1,5 \mathrm{~m}$ entre surcos y $0,8 \mathrm{~m}$ entre plantas para un total de 8.333 plantas/ha. El ensayo correspondió a un diseño experimental en bloques completos al azar (BCA) con 10 introducciones élites (tratamientos), cuatro repeticiones y cinco plantas por repetición, como unidad experimental se evaluaron cinco plantas por repetición.

Una semana antes del trasplante se realizó el control de arvenses y recolección de residuos de cosecha del cultivo anterior. Posteriormente se realizó la incorporación de 1,5 t ha ${ }^{-1}$ de materia orgánica y 1 t ha-1 de cal dolomita. Se realizó siembra en sitio definitivo a los $32 \mathrm{~d}$ después de realizado el semillero.

Para la nutrición del cultivo se tuvieron en cuenta los resultados del análisis de suelo y extracción del cultivo, al momento de la preparación del terreno. Se fraccionó la fertilización edáfica en dos aplicaciones, la primera al momento del trasplante aplicando $20 \mathrm{~g} /$ planta de la fuente 10 20-20 + 2 g/planta de micorrizas, y la segunda aplicación con mezcla de 2,4 g/planta (10-20-20) $+5,9 \mathrm{~g} /$ planta $(\mathrm{KCl})+11,8 \mathrm{~g} /$ planta (urea) + $5,9 \mathrm{~g} /$ planta $\left(\mathrm{KNO}_{3}\right)$.

Se usó riego por goteo, con distancia entre goteros de $30 \mathrm{~cm}$ y con aforo promedio por gotero de $30 \mathrm{~cm}^{3} \mathrm{~min}^{-1}$, aplicando lámina de riego de acuerdo con las fases fenológicas del cultivo, ini- 
ciando en las primeras semanas para cada planta con $0,2 \mathrm{~L} \mathrm{~d}^{-1}$ y terminado con $1,5 \mathrm{~L} \mathrm{~d}^{-1}$.

Se usó tutorado a tres ejes, instalando desde la tercera semana después del trasplante (semana tres - primer eje, semana cinco - segundo eje y semana siete - tercer eje). A medida que la planta fue creciendo semanalmente se guio al hilo tutor, se deschuponaba y deshojaba. El corte de la yema apical de las plantas se realizó cuando estas llegaron a la altura del hilo tutor (2,2 $\mathrm{m}$ de altura).

La cosecha inició a los 95 d después del trasplante, retirando el fruto del pedúnculo dejando el cáliz. El índice de cosecha utilizado fue el grado de madurez de los frutos superior al 90\%. La recolección se hizo una vez por semana durante 112 d para un total de 17 pases en el ciclo productivo.

Para el análisis de costos de producción se llevó registro de las labores agronómicas y se evaluó la eficiencia, teniendo en cuenta el tiempo ejecutado para cada labor. Todos los valores en el cálculo del costo de producción se cotizaron en Manizales, Caldas, en el cuarto trimestre de 2014. Como referencia para el costeo se usaron precios del mercado en Manizales para el cuarto trimestre de 2014. El precio de referencia para el cálculo de la relación beneficio/costo $(\mathrm{R} B / \mathrm{C})$ se realizó con el precio promedio por $\mathrm{kg}$ en los mercados de la región en los últimos 3 años (2012, 2013 y 2014).

Para la definición de los costos de producción, se trabajó con el concepto de costo operacional (Hoffmann et al., 1987), que incluye todos los costos de producción, sin tener en cuenta los intereses del capital invertido. Así, fue posible conseguir los costos de producción y los flujos de efectivo, sobre la base de las cuales se estimó la rentabilidad del cultivo, orientando el producto al mercado local.

Para la caracterización de los costos de producción se llevaron registros en una hoja de cálculo de costos adoptado del modelo de la Corporación Colombia Internacional (SIPSA, 2014). Se calcularon todos los valores en dólares americanos por hectárea (US\$ ha-1) con la tasa de cambio reportada en el cuarto trimestre de 2014 de acuerdo con el Banco de la República de Colombia.

En las estimaciones de los costos de producción, el mismo nivel tecnológico se consideró, manteniendo la proporcionalidad de horas pasadas con el trabajo manual y la cantidad de insumos. Los coeficientes técnicos (horas hombre-día y la cantidad de insumos) se basaron con las eficiencias de los trabajadores de la Granja Tesorito. Los costos se dividen en dos partes de la siguiente manera:

Operaciones manuales: se consideró el valor promedio de US\$11,68 por día de servicio (hombredía), equivalente a la remuneración pagada a los trabajadores rurales en la región, sin incluir las contribuciones a la seguridad social, ya que, en general, la actividad es realizada por mano de obra familiar o contratada en temporadas específicas.

Insumos: se usó el precio medio entre los principales distribuidores en la región.

Después de ser definidos los costos de producción (tabla 1), se estimó el flujo de caja, considerando una inversión de ocho meses. Los valores se expresan en dólares americanos por hectárea en el tiempo de la inversión.

Para analizar la rentabilidad del cultivo, se calcularon los siguientes indicadores financieros: ingresos brutos, ingresos netos, costos de producción y los indicadores económicos por cada introducción como el margen unitario de producción, relación beneficio/costo, tasa de retorno (MUP, RB/C y la TR) y se realizó un análisis de sensibilidad.

Se evaluaron los componentes del rendimiento como número de racimos por planta, rendimientos (g planta y $\mathrm{kg} \mathrm{ha}^{-1}$ ) y cosecha total. Los datos obtenidos fueron evaluados mediante análisis de varianza usando el programa estadístico SAS, versión 9.0 (SAS Inst. Inc. Cary, NC), adicionalmente se realizaron pruebas de promedios comparativos por medio del test de Duncan a nivel de significancia del $5 \%$. 
Tabla 1. Estructura de costos de producción en US $\$ \mathrm{ha}^{-1}$ de 10 introducciones élite de tomate tipo cereza en condiciones semicontroladas.

\begin{tabular}{|c|c|c|c|c|c|c|c|c|c|c|c|c|}
\hline \multirow{3}{*}{ Concepto } & \multicolumn{12}{|c|}{ Introducciones élites de tomate tipo cereza } \\
\hline & \multicolumn{2}{|c|}{ Sweet million } & \multicolumn{2}{|c|}{ IAC391 } & \multicolumn{2}{|c|}{ IAC1621 } & \multicolumn{2}{|c|}{ IAC1688 } & \multicolumn{2}{|c|}{ LA2076 } & \multicolumn{2}{|c|}{ IAC445 } \\
\hline & V/R (US\$) & $\%$ part. & V/R (US\$) & $\%$ part. & V/R (US\$) & $\%$ part. & V/R (US\$) & $\%$ part. & V/R (US\$) & $\%$ part. & V/R (US\$) & $\%$ part. \\
\hline Mano de obra & $11.182,55$ & 40,09 & $11.182,28$ & 43,79 & $11.182,27$ & 43,93 & $11.181,61$ & 44,68 & $11.184,01$ & 47,42 & $11.183,85$ & 48,32 \\
\hline Fertilizantes & 510,45 & 1,83 & 510,72 & 2,00 & 511,64 & 2,01 & 510,53 & 2,04 & 511,79 & 2,17 & 511,51 & 2,21 \\
\hline Fungicidas & 457,46 & 1,64 & 457,10 & 1,79 & 458,19 & 1,80 & 457,98 & 1,83 & 457,55 & 1,94 & 455,96 & 1,97 \\
\hline Insecticidas & 962,33 & 3,45 & 962,71 & 3,77 & 962,19 & 3,78 & 963,50 & 3,85 & 962,27 & 4,08 & 962,85 & 4,16 \\
\hline Biológicos & 560,66 & 2,01 & 561,80 & 2,20 & 560,00 & 2,20 & 560,58 & 2,24 & 561,32 & 2,38 & 560,12 & 2,42 \\
\hline Otros & $2.502,06$ & 8,97 & $2.502,54$ & 9,80 & $2.502,20$ & 9,83 & $2.502,60$ & 10,00 & $2.502,37$ & 10,61 & $2.504,33$ & 10,82 \\
\hline Subtotal & $16.178,30$ & 58,00 & $16.177,16$ & 63,35 & $16.179,04$ & 63,56 & $16.179,30$ & 64,65 & $16.179,31$ & 68,60 & $16.178,62$ & 69,90 \\
\hline Amortización & $4.287,25$ & 15,37 & $4.284,97$ & 16,78 & $4.284,03$ & 16,83 & $4.284,45$ & 17,12 & $4.283,04$ & 18,16 & $4.281,90$ & 18,50 \\
\hline Cosecha & $6.783,73$ & 24,32 & $5.074,03$ & 19,87 & $4.994,22$ & 19,62 & $4.564,74$ & 18,24 & $3.122,65$ & 13,24 & $2.684,86$ & 11,60 \\
\hline Semilla & 647,13 & 2,32 & 0,00 & 0,00 & 0,00 & 0,00 & 0,00 & 0,00 & 0,00 & 0,00 & 0,00 & 0,00 \\
\hline Total & $27.893,63$ & 100,00 & $25.536,16$ & 100,00 & $25.454,75$ & 100,00 & $25.025,98$ & 100,00 & $23.585,00$ & 100,00 & $23.145,38$ & 100,00 \\
\hline
\end{tabular}

Continúa en la siguiente tablą

\begin{tabular}{|c|c|c|c|c|c|c|c|c|c|c|}
\hline \multirow{3}{*}{ Concepto } & \multicolumn{10}{|c|}{ Introducciones élites de tomate tipo cereza } \\
\hline & \multicolumn{2}{|c|}{ IAC 426} & \multicolumn{2}{|c|}{ IAC424 } & \multicolumn{2}{|c|}{ IAC1624 } & \multicolumn{2}{|c|}{ LA2692 } & \multicolumn{2}{|c|}{ IAC412 } \\
\hline & V/R (US\$) & $\%$ part. & V/R (US\$) & $\%$ part. & V/R (US\$) & $\%$ part. & V/R (US\$) & $\%$ part. & V/R (US\$) & $\%$ part. \\
\hline Mano de obra & $11.181,75$ & 48,55 & $11.183,66$ & 49,00 & $11.183,19$ & 49,03 & $11.182,77$ & 50,46 & $11.182,31$ & 51,15 \\
\hline Fertilizantes & 511,30 & 2,22 & 511,25 & 2,24 & 510,92 & 2,24 & 511,93 & 2,31 & 511,57 & 2,34 \\
\hline Fungicidas & 458,32 & 1,99 & 458,76 & 2,01 & 458,46 & 2,01 & 458,75 & 2,07 & 456,91 & 2,09 \\
\hline Insecticidas & 962,71 & 4,18 & 963,16 & 4,22 & 962,53 & 4,22 & 961,82 & 4,34 & 961,92 & 4,40 \\
\hline Biológicos & 561,97 & 2,44 & 561,47 & 2,46 & 561,10 & 2,46 & 560,69 & 2,53 & 561,85 & 2,57 \\
\hline Otros & $2.503,51$ & 10,87 & $2.503,77$ & 10,97 & $2.502,13$ & 10,97 & $2.504,27$ & 11,30 & $2.503,18$ & 11,45 \\
\hline Subtotal & $16.177,26$ & 70,24 & $16.177,51$ & 70,88 & $16.178,34$ & 70,93 & $16.178,01$ & 73,00 & $16.177,73$ & 74,00 \\
\hline Amortización & $4.281,54$ & 18,59 & $4.279,46$ & 18,75 & $4.283,51$ & 18,78 & $4.281,63$ & 19,32 & $4.282,73$ & 19,59 \\
\hline Cosecha & $2.570,30$ & 11,16 & $2.362,26$ & 10,35 & $2.347,03$ & 10,29 & $1.699,80$ & 7,67 & $1.401,34$ & 6,41 \\
\hline Semilla & 0,00 & 0,00 & 0,00 & 0,00 & 0,00 & 0,00 & 0,00 & 0,00 & 0,00 & 0,00 \\
\hline Total & $23.031,40$ & 100,00 & $22.823,80$ & 100,00 & $22.808,88$ & 100,00 & $22.161,66$ & 100,00 & $21.861,79$ & 100,00 \\
\hline
\end{tabular}

\section{RESULTADOS Y DISCUSIÓN}

Los costos de producción para una hectárea para cada una de las introducciones élites fueron similares hasta el inicio de la cosecha, debido a que tanto las labores culturales como los insumos utilizados presentan costos semejantes. La principal variación se dio en el rubro mano de obra, específicamente asociado a las labores de la cosecha, debido a la fluctuación de la productividad que presentan cada uno de los materiales evaluados (tabla 1). Para la estimación de los cálculos de los costos totales por hectárea se tuvieron en cuenta: costos de mano de obra, costo de amortización del invernadero por un ciclo de los cuatro ciclos de vida útil, insumos, para el hibrido Sweet million la semilla tuvo un valor de US\$647,13 y para las semillas de las 10 introducciones élites no represento ningún valor puesto que se obtuvieron de bancos de germoplasma a nivel nacional. 
El costo más alto de producción lo tiene el híbrido Sweet million con US $\$ 27.893,63$ por ha, la participación de la cosecha en los costos fue alto con el $24,32 \%$ de los costos totales, debido a su alto rendimiento con $41.665 \mathrm{~kg} \mathrm{ha}^{-1}$ (tabla 2), seguido de las introducciones IAC391, IAC1621 y IAC1688 con participaciones del $19,87 \%, 19,62 \%$ y $18,24 \%$ de los costos totales respectivamente (tabla1). Las introducciones LA2692 y IAC412 fueron las de menor porcentaje de participación en mano de obra de la cosecha en los costos totales con $7,67 \%$ y $6,41 \%$, respectivamente, fundamentalmente por sus bajos rendimientos con $10.441 \mathrm{~kg} \mathrm{ha}^{-1}$ y 8.600 $\mathrm{kg} \mathrm{ha}^{-1}$, respectivamente (tabla 2). Es de resaltar la introducción IAC391 que presentó el valor más alto de la cosecha con US\$5.074,03 de las introducciones evaluadas (tabla 1). Testa et al. (2014), obtuvieron un costo de producción de tomate tipo cereza cultivado en 30 invernaderos en media de US\$229.570.

El rubro con mayor participación en los costos de producción para todos los materiales evaluados como en el testigo, fue la mano de obra la cual osciló entre 51,15\% y 40,09\% del total de los costos de producción. Destacándose con los más bajos porcentajes de participación las introducciones IAC391, IAC1621, IAC1688 con 43,79\%, $43,93 \%$ y $44,69 \%$, respectivamente. De la mano de obra, las labores culturales son las que tienen mayor participación debido a las condiciones de pendiente del terreno ( $\geq 5 \%$ ) presente en la zona (Obando et al., 2006), haciendo que cada una de las labores se realicen de forma manual.

Los costos de amortización por ciclo de 8 meses de invernadero varió entre 15,37\% y 19,59\% del total de los costos, ocupando así el segundo lugar de mayor peso en los costos. Por último los porcentajes de participación de las introducciones élites tienen un comportamiento ascendente en los costos de producción como la mano de obra, amortización del invernadero, insumos y un comportamiento descendente para la cosecha, teniendo en cuenta que esta es más concentrada y a su vez presenta un menor rendimiento que el testigo. En tanto, los mayores costos de producción en estos materiales se deben a la exigencia alta de mano de obra para las labores de tutorado y cosecha, principalmente.

En un estudio realizado por Perilla et al. (2011), con el objetivo de caracterizar las condiciones sociales, técnicas y económicas del sistema de producción de tomate bajo invernadero en los municipios de Guateque, Sutatenza y Tenza, del departamento de Boyacá (Colombia), destacan que el costo total de mano de obra, por concepto de mantenimiento del cultivo, alcanza los US\$13.821 por ha, para nuestro estudio el mantenimiento alcanzó valores de US\$11.693 por ha. Estos mismos autores resaltan a la cosecha como la operación del proceso de producción que implica mayor costo, ya que se realiza dos veces por semana y en ella se utiliza una cantidad considerable de jornales similar a la desarrollada en este estudio, ascendiendo a US\$11.182/ ha. Igualmente le sigue en costo la poda de mantenimiento, la que se realiza cada semana y se utiliza en promedio de tres a cuatro jornales. Resultados similares obtuvieron Testa et al. (2014), evaluando la producción de tomate tipo cereza en 30 invernaderos en Italia.

De la evaluación de los componentes del rendimiento se observaron diferencias significativas $(P \leq 0,05)$ conforme a la tabla 2. Para el comportamiento de la variable número de racimos por planta (NRP), sobresale la introducción IAC426 con 38 racimos/planta, seguido por Sweet million e IAC1688 con 37 y 34 racimos por planta, respectivamente. Entretanto, las introducciones IAC1621 y LA2692 tuvieron un comportamiento medio con valor de 27 racimos/planta, en tanto las introducciones LA2076, IAC424, IAC391 y IAC412 mostraron resultados de 26, 25, 24 y $20 \mathrm{racimos} / \mathrm{planta}$, respectivamente. En contraste, las introducciones IAC426 e IAC412 fueron las de peor comportamiento con diferencia cercana al $50 \%$ de 19 racimos/planta en promedio con las de mejor comportamiento (tabla 2). 
Tabla 2. Promedios de los componentes de rendimiento de 10 introducciones élite de tomate tipo cereza bajo condiciones semicontroladas.

\begin{tabular}{|l|l|l|l|l|l|l|l|}
\hline $\begin{array}{c}\text { Introducciones } \\
\text { élites }\end{array}$ & $\begin{array}{c}\text { RtoB } \\
\text { (g/planta) }\end{array}$ & PFP & $\begin{array}{c}\text { RtoN } \\
(\mathrm{g} / \text { planta })\end{array}$ & $\begin{array}{c}\text { Rto } \\
\left(\mathrm{kg} \mathrm{ha}^{-1}\right)\end{array}$ & NFR & NRP & PProF \\
\hline Sweet million & $5.123,7 \mathrm{a}$ & $124 \mathrm{a}$ & $5.000,0 \mathrm{a}$ & $41.665 \mathrm{a}$ & $14,57 \mathrm{a}$ & $37,00 \mathrm{a}$ & $8,03 \mathrm{~b}$ \\
\hline IAC391 & $3.797,9 \mathrm{~b}$ & $58 \mathrm{bc}$ & $3.740,1 \mathrm{~b}$ & $31.165 \mathrm{~b}$ & $8,30 \mathrm{bcd}$ & $24,75 \mathrm{bc}$ & $11,14 \mathrm{a}$ \\
\hline IAC1621 & $3.740,4 \mathrm{~b}$ & $61 \mathrm{bc}$ & $3.679,7 \mathrm{~b}$ & $30.665 \mathrm{~b}$ & $11,20 \mathrm{~b}$ & $27,07 \mathrm{~b}$ & $8,06 \mathrm{~b}$ \\
\hline IAC1688 & $3.448,5 \mathrm{~b}$ & $85 \mathrm{~b}$ & $3.363,9 \mathrm{~b}$ & $28.032 \mathrm{~b}$ & $15,31 \mathrm{a}$ & $34,19 \mathrm{a}$ & $5,35 \mathrm{~cd}$ \\
\hline LA2076 & $2.326,2 \mathrm{c}$ & $24 \mathrm{de}$ & $2.301,9 \mathrm{c}$ & $19.192 \mathrm{c}$ & $5,22 \mathrm{de}$ & $26,22 \mathrm{bc}$ & $7,04 \mathrm{~b}$ \\
\hline IAC445 & $1.999,9 \mathrm{~cd}$ & $22 \mathrm{de}$ & $1.978,3 \mathrm{~cd}$ & $16.482 \mathrm{~cd}$ & $5,64 \mathrm{de}$ & $18,86 \mathrm{c}$ & $7,58 \mathrm{~b}$ \\
\hline IAC426 & $1.915,9 \mathrm{~cd}$ & $22 \mathrm{de}$ & $1.893,5 \mathrm{~cd}$ & $15.774 \mathrm{~cd}$ & $10,00 \mathrm{bc}$ & $37,94 \mathrm{a}$ & $4,53 \mathrm{~d}$ \\
\hline IAC424 & $1.789,6 \mathrm{cde}$ & $48 \mathrm{~cd}$ & $1.741,3 \mathrm{cde}$ & $14.507 \mathrm{cde}$ & $14,71 \mathrm{a}$ & $26,18 \mathrm{bc}$ & $2,85 \mathrm{e}$ \\
\hline IAC1624 & $1.743,3 \mathrm{cde}$ & $14 \mathrm{e}$ & $1.729,6 \mathrm{cde}$ & $14.416 \mathrm{cde}$ & $6,67 \mathrm{de}$ & $24,47 \mathrm{bc}$ & $6,76 \mathrm{bc}$ \\
\hline LA2692 & $1.275,1 \mathrm{de}$ & $22 \mathrm{de}$ & $1.253,2 \mathrm{de}$ & $10.441 \mathrm{de}$ & $7,53 \mathrm{cde}$ & $26,82 \mathrm{~b}$ & $2,57 \mathrm{e}$ \\
\hline IAC412 & $1.106,1 \mathrm{e}$ & $74 \mathrm{bc}$ & $1.032,4 \mathrm{e}$ & $8.600 \mathrm{e}$ & $4,65 \mathrm{e}$ & $19,65 \mathrm{bc}$ & $11,88 \mathrm{a}$ \\
\hline
\end{tabular}

RtoB $=$ rendimiento en $\mathrm{g} /$ planta; PFP $=$ peso de frutos perdidos, RtoN = rendimiento neto en $\mathrm{g} /$ planta, Rto = rendimiento en $\mathrm{kg}_{\text {ha }}{ }^{-1}$, $\mathrm{NFR}=$ número de frutos por racimo, NRP = número de racimos por planta, PProF $=$ peso promedio de frutos.

Promedios con letras distintas indican diferencia significativa según la prueba de Duncan $(P \leq 0,05)$.

Para la variable número de frutos/racimo (NFR), sobresalen dos introducciones élites IAC1688 e IAC424, ambas con 15 frutos/racimo, seguido del híbrido Sweet million con 14 frutos/racimo. Comportamiento contrario fue para las introducciones LA 2076 y IAC412 con valores más bajos de cinco frutos por racimo. Para el peso promedio de fruto (PproF), las introducciones élite IAC412 e IAC391 obtuvieron 11,88 y 11,14 g/fruto, respectivamente, superior a las demás introducciones y al híbrido Sweet million que obtuvo 8,03 $\mathrm{g} /$ fruto, encontrándose dentro de los pesos exigidos por el mercado del tomate tipo cereza que es entre 8 y 15 g/fruto (Ceballos-Aguirre et al., 2012). Con pesos de frutos inferiores las introducciones LA2692, IAC424, IAC426 se hacen económicamente inviables, ya que no satisfacen las necesidades de los mercados (tabla 2).

El comportamiento de los rendimientos (RtoB) fue superior para el híbrido Sweet million con $5.000 \mathrm{~g} /$ planta y $41.665 \mathrm{~kg} \mathrm{ha}^{-1}$, presentando diferencias significativas $(P \leq 0,05)$ respecto a las 10 introducciones élites de tomate tipo cereza.
Las introducciones IAC391, IAC1621 e IAC1688 fueron las de mejores rendimientos con 3.740,1 $\mathrm{g} /$ planta y $31.165 \mathrm{~kg} \mathrm{ha}{ }^{-1}, 3.679,7 \mathrm{~g} /$ planta y $30.665 \mathrm{~kg} \mathrm{ha}^{-1}, 3.363,9 \mathrm{~g} /$ planta y $28.032 \mathrm{~g} \mathrm{ha}^{-1}$, respectivamente. Ceballos-Aguirre et al. (2012) encontraron con las introducciones IAC426, IAC1624 y el híbrido Sweet million en condiciones de campo producciones medias de $2.055 \mathrm{~g} /$ planta. Ya Macua et al. (2006, 2008) observaron en nueve variedades de tomate cereza un rendimiento promedio de $85.780 \mathrm{~kg} \mathrm{ha}^{-1}$. Testa et al. (2014) obtuvieron $166.000 \mathrm{~kg} \mathrm{ha}^{-1}$, cultivando tomate híbrido tipo cereza en condiciones de Italia que es un alto rendimiento comparado con los de este estudio y respecto a los de otros países que se encuentran en los rangos de 129.000 a 152.000 $\mathrm{kg} \mathrm{ha}^{-1}$ (Sharma et al., 2011). Según estos autores, se han alcanzado esos altos rendimientos por el uso de riego por goteo, acolchado plástico ( $\mathrm{mulch}$ ), suelos arenosos y las condiciones climáticas de Sicilia (Italia) que otorgan un crecimiento rápido del tomate tipo cereza. Para las condiciones de la región andina colombiana Ceballos-Aguirre et al. (2012) obtuvieron producciones máximas de 
tomate tipo cereza con el hibrido Sweet million 17,1 $\mathrm{t} \mathrm{ha}^{-1} \mathrm{y}$ con las introducciones IAC426 17,0 t ha ${ }^{-1}$ e IAC1624 16,1 t ha ${ }^{-1}$ ) con una densidad de 8.333 plantas/ha.

Para las pérdidas de frutos incidieron dos factores en el sistema productivo como fue la fisiopatía conocida como agrietamiento del fruto y los daños ocasionados por el insecto pasador del fruto (Tuta absoluta Meyrick), aumentando estas pérdidas en los picos de producción. El híbrido Sweet million fue el más susceptible a estas dos adversidades con el mayor número de frutos perdidos con $124 \mathrm{~g} /$ planta en todo el ciclo del cultivo, destacándose con el mejor rendimiento neto, obteniendo un porcentaje de pérdidas del 2,42\%. La introducción élite IAC1688 obtuvo 85 $\mathrm{g} /$ planta de pérdidas ubicándose en segunda lugar con más pérdidas. Las introducciones élites IAC1624, IAC445, LA2692 e IAC426 presentaron menores pérdidas con valores de 14, 22, 22 y $22 \mathrm{~g} /$ planta, respectivamente, con promedio de $0,75 \%$ de pérdidas, lo que indica que cerca del 99\% de la producción fue comercializada.

\section{Viabilidad económica de la inversión}

El valor promedio comercial en los mercados de cadena en la ciudad de Manizales fue de US\$1,08 por kg, teniendo en cuenta que el sistema de información de precios del sector agropecuario (SIPSA) no se encuentra información de tomate tipo cereza. Según la Encuesta Nacional Agropecuaria (2014) en Colombia, en términos generales, la determinación del precio del tomate es libre teniendo en cuenta el juego de la oferta y la demanda del producto. Así, Perilla et al. (2011) relatan que el Departamento Administrativo Nacional de Estadística (DANE) es el encargado de informar sobre el comportamiento de los precios de este producto en las diversas centrales de abastos del país. La relación compra venta y la determinación de los precios del tomate producido en la región está dada por la oferta y la demanda del producto. Los precios varían día tras día y el precio de compra al productor depende del día en que se vende la cosecha.

El híbrido Sweet million arrojó los mejores ingresos netos con US $\$ 17.104,57$ por ha, seguido de las introducciones élites IAC391, IAC1621 y IAC1688 con 8.122,04; 7.663,45 y US\$5.248,58, respectivamente, reflejando un alto potencial para su producción. El comportamiento de las restantes siete introducciones élites fue con ingresos netos negativos, poco atractivo financieramente (tabla 3). Evaluando dos sistemas de siembra de tomate bajo condiciones controladas, Perilla et al. (2011) obtuvieron para el modelo de siembra en el suelo un ingreso neto de US $\$ 15.151,32$, superando a los ingresos netos obtenidos por el sistema de siembra en bolsa de US $\$ 5.485,23$, atribuyendo esta diferencia a la productividad por $\mathrm{m}^{2}$ que se tiene en cada sistema (mayor densidad de población). Testa et al. (2014) obtuvieron un ingreso neto de US $\$ 2.523,29$, cifra que pone de relieve el modesto ingreso para los agricultores en las condiciones de Sicilia (Italia). De acuerdo con los mismos autores, eso es atribuible a problemas estructurales y comerciales de las granjas de tomate tipo cereza en condiciones controladas que afectan negativamente su potencial de exportación y la competitividad, además, el pequeño tamaño de las explotaciones y su proceso de producción limitan un mayor grado de mecanización de las operaciones de cultivo, con un impacto negativo en el precio de la producción, también dificulta la introducción de innovaciones tecnológicas. En consecuencia, la comercialización de tomate está prácticamente limitada a los mercados locales y regionales, como ocurre en otras zonas de producción.

Los valores de margen unitario de producción (MUP), que se encuentren hasta el punto máximo de US\$0,89 presentan un punto de equilibrio apto para un margen de utilidad adecuado en el sistema productivo, dentro de este rango se encuentran el híbrido Sweet 
million y las introducciones élites IAC391, IAC1621 e IAC1688. Para las restantes siete introducciones élite, los valores están por encima de US\$0,89 que fue el valor percibido en el mercado, tornando estas introducciones inviables financieramente (tabla 3).

De acuerdo con la relación beneficio/costo arrojada por el análisis adoptado (tabla 3), el híbrido Sweet million es el de mayor rentabilidad, seguido de las introducciones élites IAC391, IAC1621 e IAC1688 destacándose como atractivas financieramente para un inversionista en este tipo de sistema productivo, por tanto, para las restantes introducciones no se superan las perspectivas de los inversionistas, haciendo inviable la rentabilidad del cultivo. En la evaluación de la eficiencia de la inversión (TR), el híbrido Sweet million presentó una eficiencia del $61 \%$ estrechamente relacionada con la relación beneficio/costo obtenida, seguido de las introducciones élites IAC391, IAC1621 e IAC1688 con valores positivos. Perilla et al. (2011) obtuvieron una RB/C entre 1,36 y 1,14 evaluando económicamente la siembra de tomate bajo invernadero en sus dos modalidades, tanto siembra en suelo como en bolsa, constituyendo una operación rentable, puesto que supera por amplio margen al porcentaje de retribución del dinero (costo de oportunidad) ofrecido por el sistema financiero a través de la tasa de interés de oportunidad, expresada en el indicador de referencia DTF (depósito a término fijo) a $90 \mathrm{~d}$. De esta manera, el análisis económico demuestra la fragilidad en la sostenibilidad económica del sistema, tanto por la variación de los precios del producto como en lo referente a la incidencia de los incrementos impredecibles en los costos de los insumos.

Tabla 3. Análisis económico de 10 introducciones élite de tomate tipo cereza bajo condiciones semicontroladas.

\begin{tabular}{|c|c|c|c|c|c|c|c|c|c|}
\hline $\begin{array}{c}\text { Introducciones } \\
\text { élites }\end{array}$ & $\begin{array}{l}\text { Costo de } \\
\text { producción }\end{array}$ & $\begin{array}{l}\text { Rendimiento } \\
\left(\mathrm{kg} \mathrm{ha}^{-1}\right)\end{array}$ & $\begin{array}{c}\text { Precio } \\
\text { de venta } \\
\left(\mathrm{US} \$ \mathrm{~kg}^{-1}\right)\end{array}$ & $\begin{array}{l}\text { Amortización } \\
\text { inversión } \\
\text { (US\$) }\end{array}$ & $\begin{array}{l}\text { Ingreso } \\
\text { bruto } \\
\text { (US\$) }\end{array}$ & $\begin{array}{l}\text { Ingreso } \\
\text { neto } \\
\text { (US\$) }\end{array}$ & $\begin{array}{l}\text { MUP } \\
\text { (US\$) }\end{array}$ & $\mathrm{RB} / \mathrm{C}$ & TR \\
\hline Sweet million & $\$ 27.893,63$ & 41.665 & $\$ 1,08$ & $\$ 4.283,14$ & $\$ 44.998,20$ & $\$ 17.104,57$ & $\$ 0,67$ & 1,61 & 61,29 \\
\hline IAC391 & $\$ 25.536,16$ & 31.165 & $\$ 1,08$ & $\$ 4.283,14$ & $\$ 33.658,20$ & $\$ 8.122,04$ & $\$ 0,82$ & 1,32 & 31,78 \\
\hline IAC1621 & $\$ 25.454,75$ & 30.665 & $\$ 1,08$ & $\$ 4.283,14$ & $\$ 33.118,20$ & $\$ 7.663,45$ & $\$ 0,83$ & 1,30 & 30,08 \\
\hline IAC1688 & $\$ 25.025,98$ & 28.032 & $\$ 1,08$ & $\$ 4.283,14$ & $\$ 30.274,56$ & $\$ 5.248,58$ & $\$ 0,89$ & 1,21 & 20,95 \\
\hline LA2076 & $\$ 23.585,00$ & 19.192 & $\$ 1,08$ & $\$ 4.283,14$ & $\$ 20.727,36$ & $-\$ 2.857,64$ & $\$ 1,23$ & 0,88 & $-12,18$ \\
\hline IAC445 & $\$ 23.145,38$ & 16.482 & $\$ 1,08$ & $\$ 4.283,14$ & $\$ 17.800,56$ & $-\$ 5.344,82$ & $\$ 1,40$ & 0,77 & $-23,11$ \\
\hline IAC426 & $\$ 23.031,40$ & 15.774 & $\$ 1,08$ & $\$ 4.283,14$ & $\$ 17.035,92$ & $-\$ 5.995,48$ & $\$ 1,46$ & 0,74 & $-26,01$ \\
\hline IAC424 & $\$ 22.823,80$ & 14.507 & $\$ 1,08$ & $\$ 4.283,14$ & $\$ 15.667,56$ & $-\$ 7.156,24$ & $\$ 1,57$ & 0,69 & $-31,37$ \\
\hline IAC1624 & $\$ 22.808,88$ & 14.416 & $\$ 1,08$ & $\$ 4.283,14$ & $\$ 15.569,28$ & $-\$ 7.239,60$ & $\$ 1,58$ & 0,68 & $-31,75$ \\
\hline LA2692 & $\$ 22.161,66$ & 10.441 & $\$ 1,08$ & $\$ 4.283,14$ & $\$ 11.276,28$ & $-\$ 10.885,38$ & $\$ 2,12$ & 0,51 & $-49,13$ \\
\hline IAC412 & $\$ 21.861,79$ & 8.600 & $\$ 1,08$ & $\$ 4.283,14$ & $\$ 9.288,00$ & $-\$ 12.573,79$ & $\$ 2,54$ & 0,42 & $-57,53$ \\
\hline
\end{tabular}

MUP = margen unitario de producción; $\mathrm{RB} / \mathrm{C}=$ relación beneficio/costo; $\mathrm{TR}=$ tasa de retorno. 


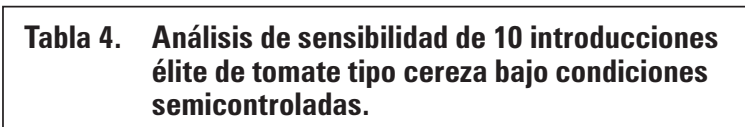

\begin{tabular}{|c|c|c|c|}
\hline Introducciones élites & MUP & $\mathrm{RB} / \mathrm{C}$ & TR \\
\hline \multicolumn{4}{|c|}{ Si el precio de venta baja $15 \%$} \\
\hline Sweet million & US $\$ 0,67$ & 1,37 & 37,09 \\
\hline IAC391 & US\$0,82 & 1,12 & 12,01 \\
\hline IAC1621 & US $\$ 0,83$ & 1,11 & 10,57 \\
\hline IAC1688 & US $\$ 0,89$ & 1,03 & 2,81 \\
\hline LA2076 & US $\$ 1,23$ & 0,75 & $-25,35$ \\
\hline IAC445 & US $\$ 1,40$ & 0,65 & $-34,64$ \\
\hline IAC426 & US\$1,46 & 0,63 & $-37,11$ \\
\hline IAC424 & US $\$ 1,57$ & 0,58 & $-41,66$ \\
\hline IAC1624 & US $\$ 1,58$ & 0,58 & $-41,99$ \\
\hline LA2692 & US $\$ 2,12$ & 0,43 & $-56,76$ \\
\hline IAC412 & US $\$ 2,54$ & 0,36 & $-63,9$ \\
\hline \multicolumn{4}{|c|}{ Si la producción disminuye $15 \%$} \\
\hline Sweet million & US $\$ 0,76$ & 1,42 & 42 \\
\hline IAC391 & US $\$ 0,94$ & 1,15 & 15 \\
\hline IAC1621 & US\$0,95 & 1,14 & 14 \\
\hline IAC1688 & US $\$ 1,02$ & 1,06 & 6 \\
\hline LA2076 & US\$1,42 & 0,76 & -24 \\
\hline IAC445 & US\$1,62 & 0,67 & -33 \\
\hline IAC426 & US\$1,69 & 0,64 & -36 \\
\hline IAC424 & US $\$ 1,82$ & 0,59 & -41 \\
\hline IAC1624 & US $\$ 1,83$ & 0,59 & -41 \\
\hline LA2692 & US\$2,47 & 0,44 & -56 \\
\hline IAC412 & US $\$ 2,96$ & 0,36 & -64 \\
\hline \multicolumn{4}{|c|}{ Disminución de la producción y el precio de venta en $15 \%$} \\
\hline Sweet million & US $\$ 0,76$ & 1,21 & 20,94 \\
\hline IAC391 & US $\$ 0,94$ & 0,98 & $-1,86$ \\
\hline IAC1621 & US\$0,95 & 0,97 & $-3,17$ \\
\hline IAC1688 & US $\$ 1,02$ & 0,90 & $-10,16$ \\
\hline LA2076 & US $\$ 1,42$ & 0,65 & $-35,26$ \\
\hline IAC445 & US $\$ 1,62$ & 0,57 & $-43,46$ \\
\hline IAC426 & US\$1,69 & 0,54 & $-45,63$ \\
\hline IAC424 & US $\$ 1,82$ & 0,50 & $-49,63$ \\
\hline IAC1624 & US $\$ 1,83$ & 0,50 & $-49,92$ \\
\hline LA2692 & US $\$ 2,47$ & 0,37 & $-62,82$ \\
\hline IAC412 & US $\$ 2,96$ & 0,31 & $-69,01$ \\
\hline
\end{tabular}

MUP = margen unitario de producción; $\mathrm{RB} / \mathrm{C}=$ relación beneficio/ costo; $\mathrm{TR}=$ tasa de retorno.
Para finalizar, el análisis de sensibilidad tiene como objetivo evaluar el impacto generado por el cambio del precio y los rendimientos del cultivo, pudiéndose determinar cuál es la introducción más rentable frente a la fluctuación del precio de venta y cantidad de frutos cosechados. De esta manera, en el caso de que el rendimiento caiga en un 15\%, el híbrido Sweet milliion y las introducciones élite IAC391, IAC1621, IAC1688, continúan siendo atractivas económicamente, ya que la relación beneficio/costo sigue siendo positiva, comportamiento similar se presenta cuando el precio de venta disminuye en un 15\% (tabla 4). Asumiendo que la producción y el precio baje en un 15\% se nota que solo el hibrido Sweet million es económicamente viable con un ingreso neto de US\$5.628,26, con un margen unitario de producción de US $\$ 0,76$ por debajo del valor de venta (US\$1,08) y una relación beneficio/costo de 1,21, lo que indica que por cada peso invertido tenemos una utilidad de 21 centavos con una eficacia de 20,94\%.

\section{CONCLUSIONES}

Según el análisis económico realizado se destacan las introducciones IAC391, IAC1621 e IAC1688 con valores superiores a uno en la relación beneficio/costo y positivos en la tasa de retorno, así como un adecuado rendimiento en las condiciones del estudio.

Los resultados del análisis económico mostraron que si los precios de venta de tomate cherry decrecen en un $15 \%$ apenas cubren los costos de producción, dejando un modesto margen de utilidad para los productores, exponiendo la fragilidad de la sostenibilidad económica del sistema, tanto por la variación de los precios del producto como en lo referente a la incidencia de los incrementos impredecibles en los costos de los insumos. 


\section{REFERENCIAS BIBLIOGRÁFICAS}

Anuario Meteorológico Cafetero. 2012 y 2013. Federación Nacional de Cafeteros de Colombia. Cenicafé, Chinchiná (Colombia).

Bai, Y. y P. Lindhout. 2007. Domestication and breeding of tomatoes: What have we gained and what can we gain in the future?. Ann. Bot. 100, 1085-1094. Doi: $10.1093 / \mathrm{aob} / \mathrm{mcm} 150$

Ceballos-Aguirre, N., F.A. Vallejo-Cabrera y N. Arango-Arango. 2012. Evaluación del contenido de antioxidantes en introducciones de tomate tipo cereza (Solanum spp.). Acta Agron. 61(3), 230-238.

Encuesta Nacional Agropecuaria. 2014. Departamento Administrativo Nacional de Estadística - DANE, En: Encuesta Nacional Agropecuaria año 2013, http://www.dane.gov.co/files/investigaciones/ agropecuario/enda/ena/2013/boletin_ena_2013. pdf; consulta: marzo de 2015.

FAOSTAT. 2013. Food and Agriculture Organization of the United Nations, Roma.

Gómez-Duque, A.M., N. Ceballos-Aguirre, F.J. OrozcoCastaño y C.A. Parra. 2010. Efecto del sistema de producción en semitecho sobre el desarrollo, rendimiento y calidad del tomate (Solanum lycopersicum L.). Agronomía (Manizales) 18(2), 47-57.

Hoffmann, R., O. Serrano, E.M. Neves, A.C. Thame y J.J. Engler. 1987. Administração da empresa agrícola. $5^{\text {a }}$ ed. Pioneira Estudos Agrícolas, São Paulo, Brasil.

Macua, J., I. Lahoz, J. Garnica, S. Calvillo, J. Zúñiga y A. Santos. 2006. Tomate de industria. Campaña 2006. Navarra Agraria 172, 19-21.
Macua, J., I. Lahoz, J. Garnica, S. Calvillo, J. Zúñiga y A. Santos. 2008. Otras variedades de tomate. Campaña 2008. Navarra Agraria. 172, 27-28.

Obando, F.H., A.M. Villegas, J.H. Betancur y L. Echeverry. 2006. Variabilidad espacial de propiedades químicas y físicas en un typic udivitrands, arenoso de la región andina central colombiana. Rev. Fac. Nal. Agr. Medellín, 59(1), 3217-3235.

Perilla, A., L.F. Rodríguez y L.T. Bermúdez. 2011. Estudio técnico-económico del sistema de producción de tomate bajo invernadero en Guateque, Sutatenza y Tenza (Boyacá). Rev. Colomb. Cienc. Hort. 5(2), 220-232. Doi: 10.17584/rcch.2011v5i2.1269

Sharma, M.K., R. Kumar y S. Kumari. 2011. Identifying superior quality F1 tomato hybrids for year round production under low cost plastic greenhouses in North-West Himalayas. Veg. Sci. 38, 30-34.

SIPSA (Sistema de Información de Precios del Sector Agropecuarios). 2014. Departamento Administrativo Nacional de Estadística - DANE. En: Sistema de Información de Precios del Sector Agropecuarios año 2014, http://www.dane.gov.co/index. $\mathrm{php} /$ agropecuario/sistema-de-informacion-deprecios-del-sector-agropecuario-sipsa; consulta: noviembre de 2014.

Testa, R., A. Trapani, F. Sgroi y S. Tudisca. 2014. Economic sustainability of italian greenhouse cherry tomato. Sustainability 6, 7967-7981. Doi: 10.3390/ su6117967 